\title{
A prospective decision-impact study incorporating Breast Cancer Index into extended endocrine therapy decision-making
}

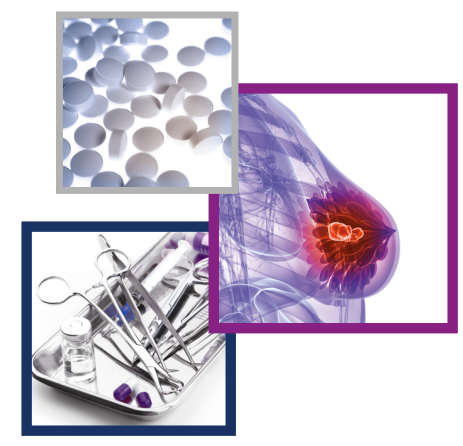

\author{
Tara Sanft*,1, Alyssa Berkowitz ${ }^{1}$, Brock Schroeder ${ }^{2}$, Christos Hatzis ${ }^{1}$, Catherine A \\ Schnabel ${ }^{2}$, Adam Brufsky ${ }^{3}$, Gary Gustavsen ${ }^{4}$, Lajos Pusztai ${ }^{1}$ \& GJ van Londen ${ }^{3}$ \\ ${ }^{1}$ Yale Cancer Center, Yale University School of Medicine, New Haven, CT 06520, USA \\ ${ }^{2}$ Biotheranostics Inc., San Diego, CA 92121, USA \\ ${ }^{3}$ University of Pittsburgh Medical Center, Pittsburgh, PA 15213, USA \\ ${ }^{4}$ Health Advances LLC, Weston, MA 02493, USA \\ *Author for correspondence: Tel.: +1 203200 2328; tara.sanft@yale.edu
}

\begin{abstract}
Aim: To prospectively assess the impact of gene expression-based assay Breast Cancer Index (BCl) on extended endocrine therapy (EET) decision-making. Patients \& methods: The BCl-tested samples from primary tumors (Stage I-III, hormone receptor positive breast cancer, $>3.5$ year endocrine therapy). Patients and physicians completed questionnaires on EET preferences and decision conflict. Using these data, a fact-based economic model was developed to project the cost impact of $\mathrm{BCl}$. Results: The $\mathrm{BCl}$ results affected treatment recommendations for 42/141 patients (overall mean, 62 year; $83 \%$ postmenopausal; $63 \%$ Stage I). Patient decision conflict decreased pre- to post-test. The $\mathrm{BCl}$-related projected net savings (US $\$ 5190 /$ patient) was robust under sensitivity analysis. Conclusion: Incorporating BCI into clinical practice meaningfully impacted physician EET recommendations and decreased patient decision conflict, with projected cost savings.
\end{abstract}

First draft submitted: 10 January 2019; Accepted for publication: 14 February 2019; Published online: 5 March 2019

Among estrogen (ER) and/or progesterone (PR) receptor-positive early-stage breast cancers, about half of recurrences occur after the first 5 years [1]. Several randomized studies have demonstrated extended endocrine therapy (EET) beyond 5 years can reduce the risk of late recurrences [2-7]. However, the absolute benefit for patients overall is a modest 3-5\% improvement in disease-free survival (DFS), whether the initial 5 years of tamoxifen (TAM) was continued for another 5 years or the therapy was switched to an aromatase inhibitor (AI) therapy. Notably, recent results from randomized control trials found that following initial adjuvant AI therapy, extended AI treatment resulted in modest DFS benefits and no overall survival (OS) benefits. This lead some in the oncology community to conclude that findings do not support the use of extended AI after 5 years although they do suggest benefit for a selected group of patients [8-10].

Previous studies of EET have shown that classic clinicopathologic factors, such as tumor size, grade, nodal status and quantitative ER, are not predictive of benefit from EET, although they may predict risk of recurrence [11-13]. Additionally, over the past decade, several gene expression profiles have been shown to be successful tools in assessing the risk of early recurrence for Stage I-II hormone receptor (HR) positive breast cancer beyond that determined by traditional clinicopathologic factors, but many of these have decreased accuracy in predicting late recurrences. The Breast Cancer Index (BCI) is a biomarker assay that is comprised of two reports, one prognostic and one predictive. The BCI prognostic and predictive assays have been validated in multiple randomized trial cohorts [14,15], have been shown to identify distinct markers of tumor proliferative status compared with tumor grade and Ki67 [16] and contain increased prognostic accuracy for late recurrence when compared with the 21-gene recurrence score $[13,17,18]$.

The goals of this study were to examine the impact of $\mathrm{BCI}$ results on physician recommendations regarding EET, to assess patient perceptions of EET before and after BCI testing and to evaluate the cost impact of BCI

Future Medicine 
testing. Preliminary results on physician decision impact and patient attitude toward EET were reported earlier based on single-institution results [19], the current report presents the final analysis of combined data from two institutions. The larger, multi-institutional results were used to develop a health economic model to assess the health economic impact of using BCI in EET decision-making. Specifically, the results from the prospective decisionimpact study allowed for modeling of comparisons between decision-making using standard clinicopathologic diagnostic modalities alone versus decision-making following incorporation of BCI. The hypothetical patient cohort was followed through the model that tracked costs across a variety of possible health states including EET or observation, recurrence and death over a 5 -year follow-up.

\section{Patients \& methods}

Prospective decision-impact study

Patients

Women with history of Stage I-III, HR+ breast cancer treated at the Yale Cancer Center or at the University of Pittsburgh Medical Center, including five practices and 17 physicians who had completed at least 3.5 years of endocrine therapy (TAM, AI or combination), were eligible for inclusion in the study. Patients were enrolled between February 2014 and April 2016. Physicians and patients completed standardized preassay and postassay questionnaires. The BCI assay results were reported to the treating physician who discussed them with the patient prior to the postassay questionnaire. This study was approved by Institutional Review Boards at both institutions, and all participants signed informed consent [19].

The physician preassay questionnaire collected baseline patient and tumor characteristics treatment history, side effects from prior endocrine therapy, an estimate of patient risk of distant recurrence, their preassay recommendation and confidence level for EET. The physician postassay questionnaire assessed the postassay recommendation for EET and level of confidence in the recommendation. The patient preassay questionnaire assessed history and severity of endocrine-therapy-related side effects, perceived risk of recurrence, preference for EET, level of concern regarding the cost, side effects, safety, potential benefit from therapy and satisfaction/comfort with the decision whether to extend endocrine therapy. The patient postassay questionnaire also assessed perceived risk, preferences, concerns and decision satisfaction, and further added the assessment of the likely impact of BCI on adherence to recommended therapy. Patients also completed the State Trait Anxiety Index and the decision conflict scale, validated instruments questionnaires designed to assess anxiety and decision conflict, respectively, before and after BCI testing [20,21].

\section{Molecular testing}

The BCI assay (Biotheranostics, Inc; CA, USA) was performed using formalin-fixed, paraffin embedded archived tumor tissue from primary tumor specimens. The BCI test report is comprised of two distinct results. The BCI Prognostic is based on the algorithmic combination of the HoxB13/IL17BR (H/I) ratio, a set of proliferation-based genes and the molecular grade index, comprised of BUB1B, CENPA, NEK2, RACGAP1 and RRM2) with four reference genes and it reports individualized risk of late distant recurrence and a risk category [15]. Notably, the BCI test report initially reported a low, intermediate or high-risk category; however, the report was modified in April 2015 to bin intermediate and high risk results into a single high risk category (based on time-dependent analysis of three validation studies $[13,15]$ showing similar late distant recurrence rates in intermediate and high risk patients). The BCI predictive component is based on the $\mathrm{H} / \mathrm{I}$ biomarker alone and reports a prediction of high versus low likelihood of benefit from EET. Based on the two distinct results, there are four possible BCI result categories (high risk-high likelihood of benefit from EET, high risk-low likelihood of benefit from EET, low risk-high likelihood of benefit from EET and low risk-low likelihood of benefit from EET).

\section{Statistical analyses}

Paired rank tests were used for comparison of pre- versus post-test patient concerns, physician confidence and patient comfort/satisfaction regarding the treatment recommendation pre- versus post-BCI testing. Paired $t$ tests were used to compare pre- versus post-test decision conflict scale and State Trait Anxiety Index questionnaires. McNemar's test for nominal paired data was used to assess change in physician treatment recommendation preversus post-BCI testing. Odds ratio (OR) was calculated using conditional logistic regression. 


\section{Health economic model}

A deterministic, decision-analytic, Microsoft Excel-based model was developed from the payer perspective to project cost and clinical outcomes of using BCI compared with standard clinicopathologic evaluation to guide ER+ breast cancer patient management for the decision whether to extend endocrine therapy from 5 to 10 years. This particular modeling approach was selected because it results in a rational and reproducible analysis despite a moderate level of technical complexity and input uncertainty [22]. The model evaluated BCI when ordered at 5 years postdiagnosis for the hypothetical population of patients, who were recurrence-free at 5 years postdiagnosis and included impact on decision-making for duration of endocrine-based therapy. Treatment of a hypothetical cohort of ER+, early-stage breast cancer patients was simulated through the development of a branched patient flow model. Branches represented patient management under two scenarios based on the results from the prospective decisionimpact study: decision-making using standard clinicopathologic diagnostic modalities alone versus decision-making following incorporation of BCI. The hypothetical patient cohort was followed through the models that tracked costs across a variety of possible health states, including EET (or no treatment), observation or recurrence and death over a 5-year follow-up.

\section{Clinical inputs}

Key assumptions driving use of EET are highlighted in Table 1. Inputs for EET decision-making were estimated based on the results of the prospective decision-impact study: pre-BCI recommendations were used for the 'without test' scenarios and post-BCI recommendations were used for the 'with test' scenarios. Pre- and postassay recommendations were averaged between institutions. The proportion of patients expected in each of the four BCI result categories based on BCI Prognostic risk of late distant recurrence and BCI predictive (H/I) likelihood of benefit from EET (high-high, high-low, low-high and low-low) were based on results provided by the test manufacturer of real-world results from 14,463 patients tested in clinical practice.

The projected impact of EET on recurrence rates was based on BCI predictive (H/I) status (high vs low) alone, derived from the analysis of $\mathrm{H} / \mathrm{I}$ in the MA.17 study cohort [14]. Baseline estimated risk of late recurrence without EET in the four BCI result categories was based on recurrence rates in placebo-treated patients with high versus low BCI predictive (H/I) from the MA.17 cohort, adjusted for prognostic risk of late distant recurrence from patients with BCI Prognostic high versus low risk in validation studies of node negative and node positive patients [15,23] and the proportion of patients in each BCI result category. The risk of recurrence was 3.1-fold higher between BCI Prognostic high and low risk patients in the High H/I group and 4.3-fold higher in the Low H/I group. Relative risk reduction from EET observed in high H/I patients from the MA.17 cohort was then applied across both BCI subgroups with high $\mathrm{H} / \mathrm{I}$. Given that patients with low $\mathrm{H} / \mathrm{I}$ did not have a statistically significant benefit from EET in the MA.17 study, no benefit was applied to these patients in the base case scenario.

\section{Adherence}

To account for the potential effect of patient adherence to endocrine therapy on the effect of therapy and the likelihood of recurrence, adherence to endocrine therapy and the associated impact on disease recurrence was modeled according to previously observed rates [24,25]. The model also incorporated the impact of a high risk BCI score on patient adherence, estimated based on a published meta-analysis evaluating improvement in adherence following information interventions [26,27]. Adherence assumptions are highlighted in Table 1 .

\section{Costs}

A variety of sources were used to build cost assumptions applied to patients as they moved through health states within the models (Table 1). Adjuvant endocrine drug costs were based on a RED BOOK analysis of average sale prices across various manufacturers, factoring in generic drug pricing for both TAM and AIs [28]. Alongside endocrine therapy, additional costs associated with supportive care and complications were included in the estimate of drug costs [29,30]. For TAM, estimated frequency and costs associated with complications including endometrial cancer, stroke, pulmonary embolism and deep vein thrombosis were included. For AIs, estimated frequency and costs associated with annual bone mineral density screening, selective bisphosphonate use for osteopenia/osteoporosis and fractures were included. Because CMS covers bone mineral density annually for women on bisphosphonate therapy, the more conservative frequency of annual bone density testing was chosen for this analysis. Follow-up and total costs of care following breast cancer recurrence were based an analysis of cumulative costs of chemotherapy, biologic use and healthcare utilization in a large insured US population (\$330,904 in patients with $25-36$ months 
Table 1. Key assumptions: clinical decision-making and compliance.

\begin{tabular}{|c|c|c|c|}
\hline \multirow{2}{*}{$\begin{array}{l}\mathrm{BCl} \text { prognostic and predictive }(\mathrm{H} / \mathrm{I}) \\
\text { results }\end{array}$} & \multirow{2}{*}{$\begin{array}{l}\text { Endocrine therapy patient flow } \\
\text { Proportion of patients }\end{array}$} & \multicolumn{2}{|c|}{ Extended endocrine therapy utilization } \\
\hline & & Without test & With test \\
\hline - High risk/high likelihood of benefit & $34 \%$ & $77 \%$ & $100 \%$ \\
\hline - Low risk/high likelihood of benefit & $9 \%$ & $54 \%$ & $43 \%$ \\
\hline - High risk/low likelihood of benefit & $21 \%$ & $75 \%$ & $69 \%$ \\
\hline - Low risk/low likelihood of benefit & $36 \%$ & $59 \%$ & $18 \%$ \\
\hline \multicolumn{4}{|l|}{ Estimated 5-year recurrence rates ${ }^{\dagger}$} \\
\hline $\begin{array}{l}\mathrm{BCl} \text { prognostic and predictive }(\mathrm{H} / \mathrm{l}) \\
\text { results }\end{array}$ & Observation & EET & \\
\hline - High risk/high likelihood of benefit & $31.5 \%$ & $12.2 \%$ & \\
\hline - Low risk/high likelihood of benefit & $10.1 \%$ & $3.9 \%$ & \\
\hline - High risk/low likelihood of benefit & $25.3 \%$ & $25.3 \%$ & \\
\hline - Low risk/low likelihood of benefit & $5.8 \%$ & $5.8 \%$ & \\
\hline \multicolumn{4}{|l|}{ Adherence impact } \\
\hline Adherence rate & Assumption & & \\
\hline - Adherent & $61 \%$ & & \\
\hline - Nonadherent & $11 \%$ & & \\
\hline - Discontinued & $28 \%$ & & \\
\hline Increased recurrence risk & Assumption & & \\
\hline - Adherent & 1.0 & & \\
\hline - Nonadherent & 1.44 & & \\
\hline - Discontinued & 1.61 & & \\
\hline Increased adherence with $\mathrm{BCl}$ & Assumption & & \\
\hline - Increase in adherence & $15 \%$ & & \\
\hline \multicolumn{4}{|l|}{ Cost assumptions } \\
\hline Adjuvant endocrine therapy costs & Yearly cost (USD) & 5-year cost (USD) & \\
\hline - Tamoxifen drug costs & 126 & 630 & \\
\hline - Tamoxifen toxicity costs ${ }^{\dagger}$ & 42 & 210 & \\
\hline - Aromatase inhibitor drug costs & 1212 & 6060 & \\
\hline - Aromatase inhibitor toxicity costs $\ddagger$ & 221 & 1105 & \\
\hline Recurrence costs & Cost (USD) & & \\
\hline - Total costs & 532,904 & & \\
\hline $\mathrm{BCl}$ test costs & Cost (USD) & & \\
\hline$-\mathrm{BCl}$ test cost & 3450 & & \\
\hline \multicolumn{4}{|c|}{$\begin{array}{l}\dagger \text { Includes cost of endometrial cancer }(0.08 \% \text { annual risk at } \$ 18,245) \text {, stroke }(0.05 \% \text { annual risk at } \$ 30,111) \text {, pulmonary embolism }(0.05 \% \text { annual risk at } \$ 16,943) \text { and deep vein } \\
\text { thrombosis }(0.05 \% \text { at } \$ 6274) \text {. } \\
\ddagger \text { Represents total cost of annual bone mineral density screening and selective bisphosphonate use for osteopenia, plus associated fractures. } \\
\text { BCl: Breast Cancer Index. }\end{array}$} \\
\hline
\end{tabular}

of follow-up available) [31]. As this analysis was published prior to the US FDA approval of palbociclib, additional costs were added to the estimate to account for the new standard of care. Recent cost-effectiveness and utility studies have reported that when compared with letrozole, the addition of palbociclib provided an additional 1.23.0 quality-adjusted life years at an incremental cost of approximately $\$ 160,000-190,000$ [32,33]. Finally, the cost of the assay was based on the manufacturer's suggested retail pricing.

\section{Analyses}

Impact on total cost was evaluated based on effect from EET-based decision-making and impact on adherence, recurrence rates and drug toxicities. Univariate sensitivity analyses were conducted to evaluate the impact of parameter input uncertainty on model outcomes.

Probabilities and cost parameters were varied over a range of clinically relevant conservative and aggressive estimates from the literature and expert elicitation. 


\section{Results}

Decision impact study

Patient \& tumor characteristics \& summary of BCI test results

A total of 155 patients were enrolled, of whom 141 completed both the pre- and post-test questionnaires (Supplementary Figure 1). The median age was 62 years (range 45-87), 117 (83\%) patients were postmenopausal, 104 (74\%) had node negative disease, 89 (63\%) had Stage 1 disease and 60 (43\%) received chemotherapy (Table 2).

'BCI Prognostic' categorized 60 patients (43\%) as high risk and 81 patients (57\%) as low risk for late distant recurrence while 'BCI predictive' categorized 60 patients (43\%) as high likelihood and 81 patients $(57 \%)$ as low likelihood of benefit from EET. In 39 cases $(28 \%)$, the assay predicted high risk of late distant recurrence and high likelihood of benefit from EET while in 60 cases $(43 \%)$, the assay predicted a low risk of late distant recurrence and low likelihood of benefit from EET. In 21 cases (15\%), the assay predicted high risk of late recurrence and low likelihood of benefit.

\section{Impact of BCI on physician recommendations for EET}

Prior to BCI testing, EET was recommended for 92 of the 141 patients (65\%). The BCI results led to a change in physician treatment recommendation in 42 patients $(30 \%$; Table 3$)$. A change from a 'recommend' to 'not recommend' EET was more common $(\mathrm{n}=30 ; 71 \%)$ than a change from 'not recommend' to 'recommend' $(\mathrm{n}=12$; 29\%; OR: 2.5; 95\% CI: 1.28-4.883; $\mathrm{p}=0.0087$ ). Overall, recommendations against EET rose from 35 to $48 \%$, while recommendations for EET dropped from 65 to $52 \%$.

Of cases in which physicians changed their recommendation from 'recommend' EET to 'not recommend' EET, $83 \%$ of patients were low risk and $75 \%$ were high risk. Additional analysis showed that $97 \%(29 / 30)$ of patients were BCI prognostic low risk or BCI predictive low likelihood of benefit. Of cases in which physicians changed their recommendation from 'not recommend' to 'recommend' EET, 92\% (11/12) were BCI Prognostic high risk or $\mathrm{BCI}$ predictive high likelihood to benefit from EET. Following BCI testing, there was an increase in physician confidence in their recommendation $(\mathrm{p}=0.017)$.

\section{Patient-reported data}

At enrollment, patients were asked to rank their concerns regarding benefit of treatment, safety, side effects and cost from 1 to $5(1=$ not at all concerned; $5=$ very much concerned $)$. Compared with baseline, post-BCI evaluation showed a significant decrease in concerns regarding benefit $(p=0.0015)$, cost $(p=0.026)$ and safety $(p<0.001)$. There was no significant change in concern for side effects of EET $(\mathrm{p}=0.262)$ from pre- to post-BCI testing.

There was a significant decrease in anxiety scores from pre- to post-testing $(31.14-29.01 ; \mathrm{p}=0.0045)$. Decision conflict significantly decreased following BCI testing $(44.8-36.3 ; \mathrm{p}<0.0001)$. Of those who reported increased anxiety, $61 \%$ had BCI results showing high likelihood of late recurrence.

Following BCI testing, there was an increase in patient satisfaction/comfort in the decision regarding EET ( $\mathrm{p}$ $<0.0001$ ). Of patients who were recommended for EET by their physician following BCI testing, $82 \%$ stated that they would be more likely to be compliant with EET based on the BCI results.

\section{Health economic analysis}

The base case resulted in mean costs of $\$ 78,850$ per patient without the use of BCI. Use of BCI resulted in mean costs of $\$ 73,660$, resulting in a savings of $\$ 5190$ per patient after accounting for the cost of the test. The cost benefit was predominantly driven by targeted use of EET.

Key outputs of the sensitivity analysis for this model are illustrated in Figure 1 . The model was most sensitive to the percentage of patients with BCI Prognostic high risk and high BCI predictive $(\mathrm{H} / \mathrm{I})$ results who received EET following $\mathrm{BCI}$ testing, the percentage of total patients who would have received EET in the absence of $\mathrm{BCI}$ testing, the benefit of EET in patients with a low BCI predictive $(\mathrm{H} / \mathrm{I})$ result and the total cost of a recurrence. None of the sensitivity analyses resulted in a cost savings under $\$ 1700$ per patient tested while more aggressive scenarios predicted cost savings of over $\$ 7300$ per patient tested.

\section{Discussion}

This study now combines data from two institutions, helping to improve generalizability and reduce bias that may be associated with specific practices at any one institution. The results from this study show incorporating results from the $\mathrm{BCI}$ test into decisions surrounding use of EET led to significant changes in physician treatment 
Short Communication Sanft, Berkowitz, Schroeder et al.

Table 2. Baseline characteristics.

Patient and tumor characteristics

$n=141$

Age, $y$

- Mean/median

$62 / 63 y$

- (Min-max)

(45-87y)

$\geq 50$ years old, $n(\%)$

$129(91 \%)$

Race, n (\%)

- Caucasian

$87(62 \%)$

- African-American

$5(4 \%)$

- Asian

$2(1 \%)$

- Unknown

$47(33 \%)$

Menopausal status, n (\%)

- Premenopausal

$17(12 \%)$

- Perimenopausal

$7(5 \%)$

- Postmenopausal

$117(83 \%)$

Hormone receptor status, n (\%)

$-\mathrm{ER}+/ \mathrm{PR}+$

$126(89 \%)$

$-\mathrm{ER}+/ \mathrm{PR}-$

$14(10 \%)$

- ER- /PR+

$1(1 \%)$

HER2 status, n (\%)

- HER2 positive

$9(6 \%)$

- HER2 negative

$130(92 \%)$

- Not available

2 (1\%)

Stage, n (\%)

- Stage I

89 (63\%)

- Stage II

$43(31 \%)$

- Stage III

$9(6 \%)$

Tumor size, n (\%)

$-\mathrm{T} 1(\leq 2 \mathrm{~cm})$

$103(73 \%)$

$-\mathrm{T} 2(>2-5 \mathrm{~cm})$

$34(24 \%)$

- T3 (>5 cm)

$4(3 \%)$

Nodal status, $\mathrm{n}(\%)$

- NO

$104(74 \%)$

- N1 (1-3 nodes)

$30(21 \%)$

- N2 (4-9 nodes)

$7(5 \%)$

Chemotherapy, n (\%)

- Yes

$60(43 \%)$

- No

$81(57 \%)$

Initial adjuvant therapy, n (\%)

- TAM

$24(17 \%)$

- Al

$93(66 \%)$

- TAM and Al

$24(17 \%)$

Bone density, n (\%)

- Normal

$42(30 \%)$

- Osteopenia

$73(52 \%)$

- Osteoporosis

$10(7 \%)$

- Not performed

$16(11 \%)$

Al: Aromatase inhibitor; ER: Estrogen; PR: Progesterone; TAM: Tamoxifen. 
Table 3. Effects of Breast Cancer Index testing on physician decisions regarding extended endocrine therapy.

\begin{tabular}{|c|c|c|}
\hline \multirow{2}{*}{$\begin{array}{l}\text { Change in treatment decision - recommend to not } \\
\text { recommend }\end{array}$} & \multicolumn{2}{|c|}{$\mathrm{H} / \mathrm{I}$ likelihood of benefit } \\
\hline & Low likelihood, $n$ & High likelihood, n \\
\hline - $\mathrm{BCl}$ low risk & $20 / 60$ & $5 / 21$ \\
\hline - $\mathrm{BCl}$ high risk & $4 / 21$ & $1 / 39$ \\
\hline \multicolumn{3}{|l|}{ Not recommend to recommend } \\
\hline - $\mathrm{BCl}$ low risk & $1 / 60$ & $2 / 21$ \\
\hline$-\mathrm{BCl}$ high risk & $3 / 21$ & $6 / 39$ \\
\hline
\end{tabular}

BCl: Breast Cancer Index; H/I: HoxB13/IL17BR

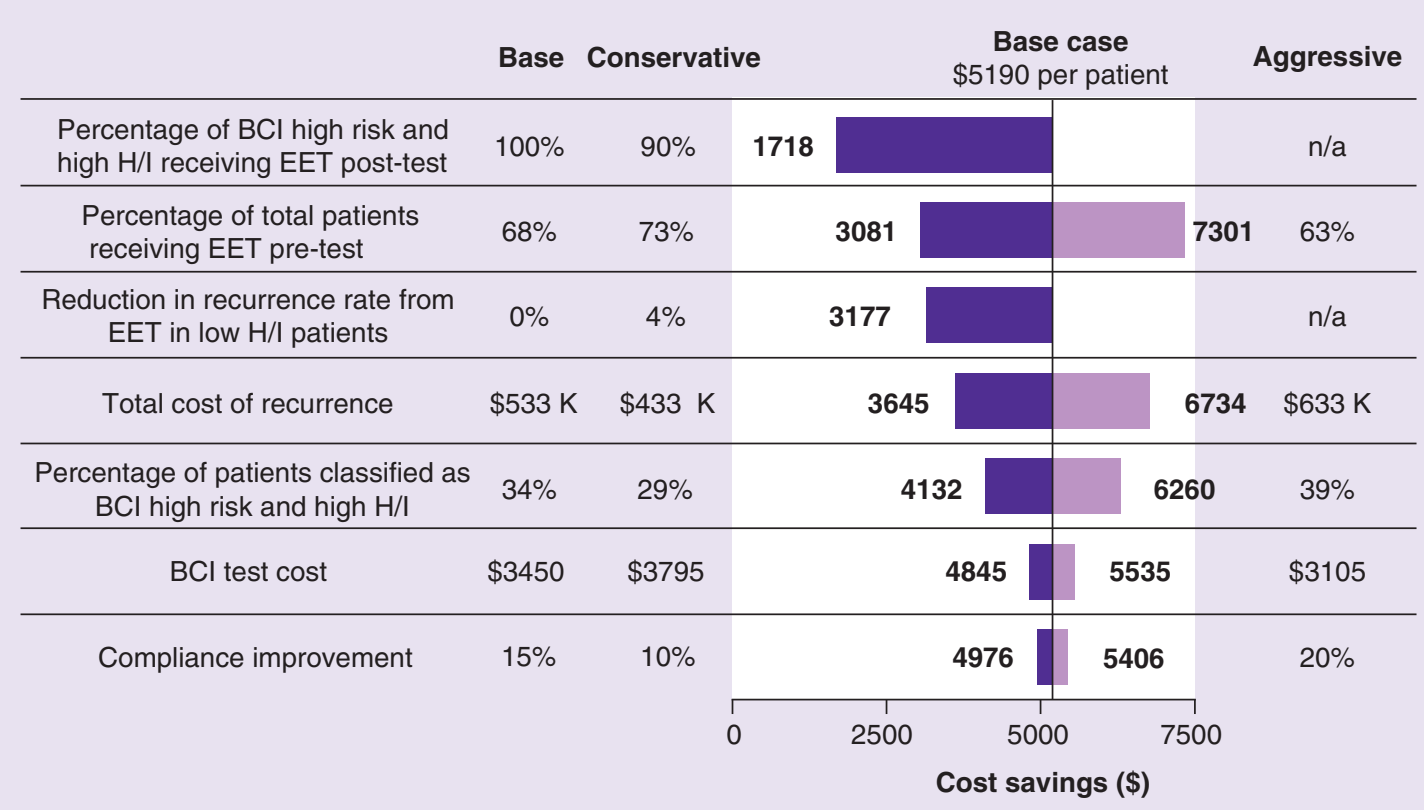

Figure 1. Univariate sensitivity analysis. The tornado diagram illustrates the sensitivity of the model to key assumptions. The cost benefit was predominantly driven by targeted use of extended endocrine therapy.

recommendations and confidence level, as well as increased patient satisfaction. The majority of changes in physician's recommendations were to omit EET after an initial recommendation to continue. The use of the BCI may have spared these patients unnecessary treatment-related side effects to prevent a recurrence that was unlikely to happen based on genomic analysis. Perhaps more impactful is the minority of patients who had a change in the recommendation to take EET after BCI test results were known. Despite the physician's clinical judgment that the patient would not benefit from EET, this group was ultimately recommended for EET, and reported increased likelihood of adherence based on the result. The goal of EET is to treat the patients most likely to benefit from prolonged therapy in order to spare them a recurrence, and this test may have helped identify those who would have otherwise been overlooked based on clinical and pathologic factors alone. Additionally, we know adherence rates continue to decline over time such that by 5 years only about $50-60 \%$ of women are compliant. Furthermore, studies in the post 5 -year setting show continued adherence issues and discontinuation rates [8,12]. Poor adherence to EET has been associated with poorer outcomes $[11,34,35]$ and has been shown to result in an increased risk of breast cancer recurrence [36], therefore helping patients manage their symptoms and encouraging drug compliance is important. Based on our results, it seems BCI could be a tool to improve adherence to EET in patients identified as high risk and high likelihood of benefit from EET. In order to better explore these implications, further chart extraction will be done over time to examine the women who were recommended to take EET and adherence rates.

When examining the potential cost impact of using BCI in women who are completing 5 years of therapy and considering EET, the test is projected to be cost-saving. Cost savings were driven predominantly by patients 
initially not recommended for EET, who were recommended for EET following BCI high risk / high likelihood to benefit results. While the proportion of patients that benefit from EET is modest in randomized studies (3-5\%), leading to challenging decision-making, the cost of a distant recurrence is significant (estimated at over $\$ 500,000$ with newer approved therapies including mTOR inhibitors and CDK 4/6 inhibitors) [31,37]. Thus, prevention of even a small number of metastatic events has the potential to greatly reduce overall cost of care. In addition, while costs from endocrine therapies themselves are relatively low, there is additional cost savings through reduction in care and therapies required for prevention and treatment of endocrine therapy-related toxicities in patients not recommended for EET following BCI testing.

Our study has several limitations. First, this study was initiated prior to the results of MA.17R, NSABP B-42, DATA and IDEAL studies were reported. Therefore, it is possible that more physicians today are not recommending EET at baseline compared with when this study was done. However, it is important to note that commentaries from these studies have identified the need for better identification of those who would benefit the most from EET, and the use of genomic platforms is recognized as one effort to better individualize recommendations. Our results do confirm that the use of BCI testing impacted decision-making and increased physician confidence. Second, there was an imbalance in the number of patients who received TAM (17\%) versus AI (66\%) as an initial adjuvant therapy. While the DFS benefit of EET appears to be in the same range for both TAM and AI (3-5\%) [2-7], the side effects and patient's perception of benefit from medication or BCI results may be impacted. A future study could examine this confound to provide further impact of initial therapy. Third, while the health economic analysis improves on a previous model [38] by incorporating real-world decision-impact, cost and recurrence assumptions are still modeled based on published literature. Future studies should confirm the cost impact using longitudinal data from third-party payer databases.

\section{Conclusion}

Extending endocrine therapy beyond 5 years in the entire early stage, ER+ breast cancer population confers only marginal benefit that can vary depending on sequencing of ET. However, specific patients do benefit greatly from EET and the identification of those patients with tools beyond clinicopathologic characteristics is important. Our multi-institutional study shows use of BCI significantly impacted physician decision-making, increased their confidence in the recommendation and led to improved patient satisfaction, increased likelihood of adherence and projected cost savings.

\section{Summary points}

- Extending endocrine therapy (EET) beyond 5 years does not appear to confer a benefit in the entire population of patients with early-stage estrogen-positive breast cancer.

- In order to identify appropriate EET patients and predict treatment benefit, additional factors outside of traditional clinicopathologic data are necessary.

- We assessed the impact of the biomarker assay Breast Cancer Index (BCI) on decision-making around EET and developed an economic model to assess cost savings.

- The $\mathrm{BCl}$ results led to a change in physician treatment recommendation for $30 \%$ of patients; in $71 \%$ of these cases, this change was from 'recommend EET' to 'not recommend EET'.

- Patient anxiety and decision conflict regarding EET significantly decreased after testing and satisfaction/comfort in the decision increased.

- Among patients who were recommended EET, $82 \%$ reported they were more likely to comply with treatment based on the $\mathrm{BCl}$ results.

- Use of $\mathrm{BCl}$ resulted in mean cost savings of US $\$ 5190 /$ patient, with the cost predominantly driven by targeted use of EET.

- Our multi-institutional study shows that the use of BCl significantly impacted physician decision-making and increased confidence and satisfaction in the decision for both the physicians and the patients. 
Financial \& competing interests disclosure

This study was funded by Biotheranostics, Inc. It should be noted that no member of Biotheranostics was involved in the data gathering or analysis of these findings. The authors have no other relevant affiliations or financial involvement with any organization or entity with a financial interest in or financial conflict with the subject matter or materials discussed in the manuscript apart from those disclosed.

No writing assistance was utilized in the production of this manuscript.

\section{Open access}

This work is licensed under the Attribution-NonCommercial-NoDerivatives 4.0 Unported License. To view a copy of this license, visit http://creativecommons.org/licenses/by-nc-nd/4.0/

\section{Conflict of interest}

T Sanft, A Berkowitz, C Hatzis and GJ van Londen declare no potential conflicts of interest. B Schroeder was an employee and shareholder in Biotheranostics, Inc. at the time the analyses were conducted. CA Schnabel is an employee and shareholder, and holds a leadership role at Biotheranostics, Inc. Adam Brufsky and Gary Gustavsen have served in advisory roles for Biotheranostics, Inc. L Pusztai has received honoraria from Biotheranostics, Inc., Celgene, Merck, and Pfizer; served in advisory roles for Celgene and Clovis Oncology; and received research funding from Foundation Medicine, Genentech, Merck, and Seattle Genetics.

Trial registration: ClinicalTrials.gov identifier NCT02057029

\section{References}

Papers of special note have been highlighted as: $\bullet$ of interest

1. Early Breast Cancer Trialists' Collaborative Group. Effects of chemotherapy and hormonal therapy for early breast cancer on recurrence and 15-year survival: an overview of the randomised trials. Lancet365(9472), 1687-1717 (2005).

2. Early Breast Cancer Trialists' Collaborative Group. Tamoxifen for early breast cancer: an overview of the randomised trials. Lancet 351(9114), 1451-1467 (1998).

3. Jordan VC, Dix C. J, Allen K E. The effectiveness of long-term treatment in a laboratory model for adjuvant hormone therapy of breast cancer. In: Adjuvant Therapy of Cancer II. Salmon SE Jones SE (Eds). Greene and Stratton, NY, USA, 19-26 (1979).

4. Jordan VC, Allen KE. Evaluation of the antitumour activity of the non-steroidal antioestrogen monohydroxytamoxifen in the DMBA-induced rat mammary carcinoma model. Eur. J. Cancer. 16(2), 239-251 (1980).

5. Goss PE, Ingle JN, Pritchard KI et al. Extending aromatase-inhibitor adjuvant therapy to 10 years. N. Engl. J. Med. 375(3), 209-219 (2016).

6. Gray RG, Rea D, Handley K et al. aTTom: long-term effects of continuing adjuvant tamoxifen to 10 years versus stopping at 5 years in 6,953 women with early breast cancer. J. Clin. Oncol. 31(18_suppl), 5 (2013).

7. Mamounas EP, Jeong JH, Wickerham DL et al. Benefit from exemestane as extended adjuvant therapy after 5 years of adjuvant tamoxifen: intention-to-treat analysis of the National Surgical Adjuvant Breast And Bowel Project B-33 trial. J. Clin. Oncol. 26(12), 1965-1971 (2008).

8. Blok EJ, Kroep JR, Meershoek-Klein Kranenbarg E et al. Optimal duration of extended adjuvant endocrine therapy for early breast cancer; results of the IDEAL trial (BOOG 2006-05). J Natl Cancer Inst. 110(1), (2018).

9. Mamounas EP, Bandos H, Lembersky BC et al. A randomized, double-blinded, placebo- controlled clinical trial of extended adjuvant endocrine therapy $(\mathrm{tx})$ with letrozole $(\mathrm{L})$ in postmenopausal women with hormone-receptor $(+)$ breast cancer $(\mathrm{BC})$ who have completed previous adjuvant tx with an aromatase inhibitor (AI): Results from NRG Oncology/NSABP B-42 [abstract]. Cancer Res. 77(4_suppl), S1-05 (2017).

10. Tjan-Heijnen V, Van Hellemond I, Peer P et al. First results from the multicenter phase III DATA study comparing 3 versus 6 years of anastrozole after 2-3 years of tamoxifen in postmenopausal women with hormone receptor-positive early breast cancer. Cancer Res. 77(4_suppl), abstr S1-03 (2017).

11. Davies C, Pan H, Godwin J et al. Long-term effects of continuing adjuvant tamoxifen to 10 years versus stopping at 5 years after diagnosis of oestrogen receptor-positive breast cancer: ATLAS, a randomised trial. Lancet 381(9869), 805-816 (2013).

12. Goss PE, Ingle JN, Martino $S$ et al. Randomized trial of letrozole following tamoxifen as extended adjuvant therapy in receptor-positive breast cancer: updated findings from NCIC CTG MA.17. J. Natl Cancer Inst. 97(17), 1262-1271 (2005).

13. Sgroi DC, Sestak I, Cuzick J et al. Prediction of late distant recurrence in patients with oestrogen-receptor-positive breast cancer: a prospective comparison of the breast-cancer index (BCI) assay, 21-gene recurrence score, and IHC4 in the TransATAC study population. Lancet Oncol. 14(11), 1067-1076 (2013).

- Breast Cancer Index (BCI) was shown to identify patients at high risk for late distant recurrence who might benefit from extended endocrine therapy (EET). 
14. Sgroi DC, Carney E, Zarrella E et al. Prediction of late disease recurrence and extended adjuvant letrozole benefit by the HOXB13/IL17BR biomarker. J. Natl Cancer Inst. 105(14), 1036-1042 (2013).

- In this validation study, BCI predictive (HoxB13/IL17BR) predicted benefit of EET and identified a subgroup of estrogen-positive patients, who were disease free after 5 years of tamoxifen and were at risk of late recurrence, and also predicted benefit from EET.

15. Zhang Y, Schnabel CA, Schroeder BE et al. Breast cancer index identifies early-stage estrogen receptor-positive breast cancer patients at risk for early- and late-distant recurrence. Clin. Cancer Res. 19(15), 4196-4205 (2013).

- This validation study demonstrated that BCI has clinical use for decisions of chemotherapy at diagnosis and for EET beyond 5 years.

16. Soliman H, Schroeder BE, Zhang Y, Magliocco AM, Schnabel CA. Correlation of breast cancer index (BCI) risk classification with tumor grade and Ki-67 in a large series of patients with early-stage, ER+ breast cancer [abstarct]. Cancer Res. 77(4_suppl), abstr P2-05-19 (2017).

17. Sestak I, Zhang Y, Schroeder BE et al. Cross-stratification and differential risk by Breast Cancer Index and recurrence score in women with hormone receptor-positive lymph node-negative early-stage breast cancer. Clin. Cancer Res. 22(20), 5043-5048 (2016).

18. Sestak I, Buus R, Cuzick J et al. Comparison of the performance of 6 prognostic signatures for estrogen receptor-positive breast cancer: a secondary analysis of a randomized clinical trial. JAMA Oncol. 4(4), 545-553 (2018).

- For women with node-negative disease, BCI, Prosigna risk of recurrence and EndoPredict (EP-clin) were significantly prognostic for late distant recurrence compared with Oncotype Dx recurrence score or clinical treatment score.

19. Sanft T, Aktas B, Schroeder B et al. Prospective assessment of the decision-making impact of the Breast Cancer Index in recommending extended adjuvant endocrine therapy for patients with early-stage ER-positive breast cancer. Breast Cancer Res. Treat. 154(3), 533-541 (2015).

20. O'Connor AM. Validation of a decisional conflict scale. Med. Decis. Making 15(1), 25-30 (1995).

21. Spielberger CD, Gorsuch R, Lushene RE, Vagg PR, Jacobs GA. Manual for the State-Trait Anxiety Inventory. Consulting Psychologists Press, CA, USA (1983).

22. Broekhuizen H, Groothuis-Oudshoorn CG, Van Til JA, Hummel JM, Mj IJ. A review and classification of approaches for dealing with uncertainty in multi-criteria decision analysis for healthcare decisions. Pharmacoeconomics 33(5), 445-455 (2015).

23. Zhang Y, Jerevall PL, Schroeder BE et al. Validation of a prognostic model integrating Breast Cancer Index (BCI) with tumor size and grade for prediction of distant recurrence in hormone receptor-positive (HR+) breast cancer with 1-3 positive nodes. J. Clin. Oncol. 34(15_suppl), abstr 541 (2016).

- Findings from this study indicate that integration of clinicopathologic and molecular factors with BCI can inform treatment decisions for node-positive patients.

24. Hershman DL, Shao T, Kushi LH et al. Early discontinuation and non-adherence to adjuvant hormonal therapy are associated with increased mortality in women with breast cancer. Breast Cancer Res. Treat. 126(2), 529-537 (2011).

25. Myrick ME, Schmid SM, Kilic N, Guth U. Eligibility, compliance and persistence of extended adjuvant endocrine therapy for breast cancer. Acta Oncol. 51(2), 247-253 (2012).

26. Kripalani S, Yao X, Haynes RB. Interventions to enhance medication adherence in chronic medical conditions: a systematic review. Arch. Intern. Med. 167(6), 540-550 (2007).

27. New England Healthcare Institute. Thinking outside the pillbox: a system-wide approach to improving patient medication adherence for chronic disease. (2009). www.nehi.net/writable/publication_files/file/pa_issue_brief_final.pdf

28. RED BOOK. IBM Micromedex 2.0 (electronic version). (2019). http://www.micromedexsolutions.com

29. Noah-Vanhoucke J, Green LE, Dinh TA, Alperin P, Smith RA. Cost-effectiveness of chemoprevention of breast cancer using tamoxifen in a postmenopausal US population. Cancer 117(15), 3322-3331 (2011).

30. Younis T, Groom A. The value-for-money of adjuvant aromatase inhibitors: time to put the debate to rest? Curr. Oncol. 22(2), 77-79 (2015).

31. Ray S, Bonthapally V, Mcmorrow D, Bonafede M, Landsman-Blumberg P. Patterns of treatment, healthcare utilization and costs by lines of therapy in metastatic breast cancer in a large insured US population. J. Comp. Eff. Res. 2(2), 195-206 (2013).

32. Mistry R, May JR, Suri G et al. Cost-effectiveness of ribociclib plus letrozole versus palbociclib plus letrozole and letrozole monotherapy in the first-line treatment of postmenopausal women with HR+/HER2-advanced or metastatic breast cancer: a U.S. payer perspective. J. Manag. Care Spec. Pharm. 24(6), 514-523 (2018).

33. Raphael J, Helou J, Pritchard KI, Naimark DM. Palbociclib in hormone receptor positive advanced breast cancer: a cost-utility analysis. Eur. J. Cancer 85, 146-154 (2017).

34. Makubate B, Donnan PT, Dewar JA, Thompson AM, Mccowan C. Cohort study of adherence to adjuvant endocrine therapy, breast cancer recurrence and mortality. Br. J. Cancer 108(7), 1515-1524 (2013).

35. Mccowan C, Shearer J, Donnan PT et al. Cohort study examining tamoxifen adherence and its relationship to mortality in women with breast cancer. Br. J. Cancer 99(11), 1763-1768 (2008). 
36. Barron TI, Cahir C, Sharp L, Bennett K. A nested case-control study of adjuvant hormonal therapy persistence and compliance, and early breast cancer recurrence in women with Stage I-III breast cancer. Br. J. Cancer 109(6), 1513-1521 (2013).

37. Xie J, Hao Y, Zhou ZY, Qi CZ, De G, Gluck S. Economic evaluations of everolimus versus other hormonal therapies in the treatment of HR+/HER2- advanced breast cancer from a US payer perspective. Clin. Breast Cancer 15(5), e263-276 (2015).

38. Gustavsen G, Schroeder B, Kennedy P et al. Health economic analysis of Breast Cancer Index in patients with ER+, LN- breast cancer. Am. J. Manag. Care 20(8), e302-310 (2014).

- Identifies cost savings with BCI utilization, at diagnosis or 5 years postdiagnosis, related to reduction in unnecessary treatment or increased EET compliance. 
